SUPOPRTING INFORMATION

S-1 Cover Page

Primary article:

\title{
Proteome Analysis of Rheumatoid Arthritis Gut Mucosa
}

Tue Bjerg Bennike, ${ }^{1, *}$, Torkell Ellingsen ${ }^{2,3}$, Henning Glerup ${ }^{3,4}$, Ole Kristian Bonderup ${ }^{3,4}$, Thomas Gelsing Carlsen ${ }^{5}$, Michael Kruse Meyer ${ }^{5,6}$, Martin Bøgsted ${ }^{7,8}$, Gunna Christiansen ${ }^{9}$, Svend Birkelund ${ }^{5}$, Vibeke Andersen $^{10,11, t,}$, Allan Stensballe $e^{5, t}$

${ }^{1}$ Departments of Pathology, Boston Children's Hospital and Harvard Medical School, Boston, Massachusetts 02115, United States

${ }^{2}$ Department of Rheumatology, Odense University Hospital, Odense DK-5000, Denmark

${ }^{3}$ University Research Clinic for Innovative Patient Pathways, Aarhus University, Aarhus DK- 8000, Denmark

${ }^{4}$ Diagnostic Center, Section of Gastroenterology, Regional Hospital Silkeborg, Silkeborg DK-8600, Denmark

${ }^{5}$ Department of Health Science and Technology, Aalborg University, Aalborg DK-9220, Denmark

${ }^{6}$ Department of Rheumatology, and Center for Clinical Research, North Denmark Regional Hospital, Hjoerring DK9800, Denmark

${ }^{7}$ Department of Clinical Medicine, Aalborg University, Aalborg DK-9220, Denmark

${ }^{8}$ Department of Haematology, Aalborg University Hospital, Aalborg DK-9000, Denmark

${ }^{9}$ Department of Biomedicine, Aarhus University, Aarhus DK-8000, Denmark

${ }^{10}$ Institute of Regional Health Research-Center Soenderjylland, University of Southern Denmark, Odense DK-5230, Denmark

${ }^{11}$ Molecular Diagnostic and Clinical Research Unit, Hospital of Southern Jutland, Aabenraa DK-6200, Denmark

${ }^{*}$ To whom correspondence should be addressed: Tue Bjerg Bennike, Departments of Pathology, Boston Children's Hospital and Harvard Medical School, Boston, Massachusetts 02115, United States. Email:

Tue.Bennike@childrens.harvard.edu

Table of Contents:

S-1: Cover page (.docx)

S-2: Colon Mucosa Proteins (.xls) Cumulated list of colon mucosa proteins.

S-3: Citrullinated peptides (.xls) List of colon mucosa peptides. 